\title{
openheart Left ventricular ejection fraction adds value over the GRACE score in prediction of 6-month mortality after ACS: the MADDEC study
}

\author{
Nina Syyli, ${ }^{\oplus 1}$ Markus Hautamäki, ${ }^{1}$ Kari Antila, ${ }^{2}$ Shadi Mahdiani, ${ }^{2}$ Markku Eskola, ${ }^{3}$ \\ Terho Lehtimäki, ${ }^{1,4,5}$ Kjell Nikus, ${ }^{1,3,5}$ Leo-Pekka Lyytikäinen, ${ }^{1,3,4,5}$ Niku Oksala, ${ }^{1,5,6}$ \\ Jussi Hernesniemi ${ }^{1,3,5}$
}

\begin{abstract}
- Additional material is published online only. To view please visit the journal online (http://dx.doi.org/10.1136/ openhrt-2019-001007).
\end{abstract}

To cite: Syyli N, Hautamäki M, Antila $\mathrm{K}$, et al. Left ventricular ejection fraction adds value over the GRACE score in prediction of 6-month mortality after ACS: the MADDEC study. Open Heart 2019;6:e001007. doi:10.1136/ openhrt-2019-001007

Received 6 January 2019 Revised 25 April 2019 Accepted 30 May 2019

\section{Check for updates}

\section{(c) Author(s) (or their}

employer(s)) 2019. Re-use permitted under CC BY-NC. No commercial re-use. See rights and permissions. Published by BMJ.

For numbered affiliations see end of article.

Correspondence to Nina Syyli; nina.syyli@tuni.fi

\section{ABSTRACT}

Background Reduced left ventricular ejection fraction (LVEF) is a risk marker for mortality after an acute coronary syndrome (ACS). Global Registry of Acute Coronary Events (GRACE) risk score, developed almost two decades ago, is the preferred scoring system for risk stratification in ACS. The aim of this study was to validate the GRACE score and evaluate whether LVEF has incremental predictive value over the GRACE in predicting 6-month mortality after ACS in a contemporary setting.

Methods A retrospective analysis of all 1576 consecutive patients who were admitted to Tays Heart Hospital and underwent coronary angiography for a first episode of ACS (2015-2016). Clinical risk factors were extensively recorded. Adjusted Cox regression analysis was used to analyse the associations between LVEF and the GRACE score with 6-month all-cause mortality. The incremental predictive value was assessed by the change in C-statistic by Delong's method for paired samples and by index of discrimination improvement (IDI).

Results In univariable analysis, both LVEF and the GRACE were associated with 6-month mortality, and after applying both variables into the same model, the results remained significant (GRACE score: HR: 1.036, 95\% Cl 1.030 to 1.042; LVEF: HR: $0.965,95 \%$ Cl 0.948 to 0.982 , both HRs corresponding to a one unit change in the exposure variable). The GRACE score demonstrated good discrimination for mortality (C-statistic: $0.833,95 \%$ $\mathrm{Cl} 0.795$ to 0.871 ). Adding LVEF to the model with the GRACE score improved model performance significantly (C-statistic: $0.848,95 \% \mathrm{Cl} 0.813$ to $0.883, \mathrm{p}=0.029$ for the improvement and IDI $0.0171,95 \% \mathrm{Cl} 0.0016$ to 0.0327 , $\mathrm{p}=0.031$ ).

Conclusions Adding LVEF to the GRACE score significantly improves risk prediction of 6-month mortality after ACS.

\section{INTRODUCTION}

Acute coronary syndrome (ACS) represents one of the leading causes of death in the western countries. ACS refers to a spectrum of clinical conditions ranging from ST-segment elevation myocardial

\section{Key questions}

What is already known about this subject?

- The Global Registry of Acute Coronary Events (GRACE) score performs well in prediction of 6 -month all-cause mortality in acute coronary syndrome. Left ventricular ejection fraction (LVEF) is an independent risk factor for mortality regardless of patient status at admission measured by the GRACE score.

What does this study add?

- Adding LVEF to risk evaluation with the GRACE score significantly improves accuracy in risk prediction.

How might this impact on clinical practice?

- LVEF can be used to improve risk prediction over the GRACE score.

- Furthermore, LVEF should be systematically recorded as well in electronic health records (EHRs) as the utilisation of EHR increase in the emerging era of 'big data' in cardiovascular medicine and research.

infarction (STEMI) to non-ST-segment elevation myocardial infarction (NSTEMI) and unstable angina. ${ }^{2}$ Numerous risk assessment instruments have been developed to quantify the risk of mortality among patients with ACS. ${ }^{3}{ }^{4}$ Currently, the Global Registry of Acute Coronary Events (GRACE) clinical risk score is the most frequently used and recommended. ${ }^{4-7}$

According to previous studies, reduced left ventricular ejection fraction (LVEF) is associated with an increased risk of death in ACS. ${ }^{8-10}$ LVEF was not included in the development of the GRACE score and very little is known about possible improvement of prognostic evaluation in patients with ACS by adding LVEF to the GRACE score. Based on one retrospective registry of patients treated between 2004 and 2005, LVEF seems to have uncertain or little additional value 
over the GRACE score. However, the same study also demonstrated that among patients treated by percutaneous coronary intervention (PCI), the predictive value of GRACE was significantly diminished when compared with patients treated conservatively. ${ }^{11}$

During the past few years, advances have been made in the treatment strategies and management of patients with acute myocardial infarction (MI).$^{12}$ For instance, the rates of different revascularisation modalities, such as coronary artery bypass grafting (CABG) and PCI, have increased; this is also the case for primary PCI in the management of STEMI. Similarly, LVEF-guided implantation of intracardiac defibrillators (ICD) has been introduced in the routine clinical work after the development of the GRACE score. ${ }^{13}$ Furthermore, the use of ultrasound in the assessment of LVEF has become mainstream in clinical work globally after the development of GRACE. Ultrasound is also used when assessing the need for LVEF-guided implantation of ICDs, a therapy aimed to reduce mortality in the long term.

The objectives of this study were to evaluate the predictive value of the GRACE score with respect to 6-month mortality and to explore changes in the prognostic performance of the GRACE score when adding LVEF data in a contemporary setting among consecutive patients treated for ACS in a tertiary referral hospital (years 2015 and 2016). This cohort comprises patients treated according to the most recent guidelines with almost exclusive use of primary PCI for treatment of ST-elevation infarction, predominant use of radial access for angiography, use of newer generation drug-eluting stents, use of ticagrelor as a first-line adenosine diphosphate (ADP) blocker along with all other available emerged medical therapies recommended to reduce cardiovascular mortality after ACS. ${ }^{56}$ Due to many advances that have transformed the treatment of ACS during the last decade, it is important to determine whether the risk stratification should be updated.

\section{METHODS}

\section{Study cohort}

Mass Data in Detection and Prevention of Serious Adverse Events in Cardiovascular Disease (MADDEC) is an ongoing, retrospective registry study that integrates data from several electronic sources from patients treated in a single tertiary care centre, Tays Heart Hospital, which is the sole provider of specialised cardiac care for a population of approximately 0.5 million inhabitants (hospital district of Pirkanmaa, Finland). ${ }^{14}$

In this study, we investigated patients, who underwent coronary angiography for a first episode of ACS during a 2-year period (from January 1, 2015 to December 31, 2016). During this time period, altogether 1795 patients underwent coronary angiography for suspected ACS. Patients who experienced a type 2 MI, or who were diagnosed with some other condition than ACS, were excluded from the study population $(\mathrm{n}=219)$. Accordingly, 1576 patients were included in the final analysis. Subjects $(n=163)$ who were treated for ACS (unstable angina pectoris (UAP) or type I MI) but did not undergo invasive evaluation due to poor medical overall prognosis, refusal or for other medical reasons were not included in the study. These subjects were on average almost 10 years older (78 (11) vs $69(12), \mathrm{p}<0.0001)$ and had higher prevalence of dementia $(10.5 \%$ vs $2.3 \%, \mathrm{p}<0.001)$, active malignancy $(6.8 \%$ vs $3.4 \%, \mathrm{p}=0.028)$ and history of cerebrovascular accident $(15.4 \%$ vs $8.3 \%, \mathrm{p}=0.002)$. There was no significant difference in sex distribution between those undergoing invasive evaluation and those who did not $(\mathrm{p}=0.224)$.

Due to the nature of this retrospective registry study, formal ethical approval was not required. However, the present study was approved by the local authority overseeing the use of registry data as required by Finnish legislature. This study was conducted according to the ethical principles of the Declaration of Helsinki on the use of human data.

\section{Data sources}

Clinical cardiovascular phenotype data were collected from the KARDIO registry, which includes detailed information of patients undergoing invasive operations and treated in the coronary care unit and/or at the cardiac wards. The study data were collected and added into the KARDIO registry by the treating physicians during hospitalisation. Additional information, such as patient characteristics, laboratory values and length of hospital stay, were extracted from the hospital electronic health records. Data of prescribed medications at the time of discharge, transfer to secondary healthcare provider or before death were verified by checking all written patient records individually. The data of the main outcome-6month mortality-was received from Causes of Death register, maintained by Statistic Finland, which records $100 \%$ of deaths of Finnish citizens at home and nearly $100 \%$ abroad. ${ }^{1516}$ In this study, the coverage of follow-up for mortality was $100 \%$ (ie, no loss to follow-up). All data collected was integrated into one structured query language database hosted in the PostgreSQL version 9.5.

\section{Main outcome and main exposure variables}

The main outcome in the present study was 6-month all-cause mortality, and the main exposure variables were the GRACE score and LVEF. The GRACE score was calculated for patients based on eight parameters: ST-segment deviation, age, heart rate, systolic blood pressure, creatinine value, Killip class, cardiac arrest at admission and elevated cardiac enzymes (troponin T -levels exceeding the 99th upper limit of the population $(50 \mathrm{ng} / \mathrm{L})$ on admission or within 24 hours of admission). For 35 patients $(2.2 \%)$, the calculation was performed after imputation of missing values of individual components of the GRACE score using multiple imputation by chained equations (mice package for $\mathrm{R}$ ). The echocardiographic LVEF was measured for 1556 patients $(98.7 \%)$ during 
hospitalisation. Images were obtained from parasternal and apical views by treating physicians as a part of routine clinical evaluation. LVEF was usually measured from multiple views combining information from all areas of the left ventricle and the mean value of all measurements was combined to form a global estimate of LVEF. After the ultrasound, physicians recorded the measurement to the KARDIO registry. If the measurement was not recorded online, the results of the ultrasound were later retrieved from written records and updated to the registry. Imputation was used to estimate the missing 20 values (1.3\%).

\section{Statistical analysis}

Cox regression analysis was used to examine the association between the GRACE score and LVEF with prediction of 6-month mortality. The risk associated with the GRACE score and LVEF is reported as the hazard ratio (HR) related to a one-unit increase in the exposure variables. Testing of the proportional hazards assumption was based on the correlation of survival rankings with Schoenfeld residuals. All variables fulfilled this assumption. Successful model calibration for all models was verified with the Hosmer-Lemeshow test. The predictive value of different models was estimated by calculating C-statistic from the receiving operating characteristics (ROC) curves using predicted risk values for 6-month mortality. The incremental predictive value of adding LVEF to the GRACE score prediction was assessed by the significance of the change in C-statistic by the DeLong's method for paired samples (for same subjects of the same study population). The incremental predictive value was also assessed by the integrated discrimination improvement (IDI) depicting the average change in predicted risk between individuals with and without the outcome after including LVEF into the risk prediction and with continuous net reclassification index (NRI). A p value of 0.05 or below was considered statistically significant. All analyses were performed using IBM SPSS Statistics (V.21.0) and R-software (packages mice, miceadds, PredictABEL and pROC).

\section{RESULTS}

\section{Baseline demographics and mortality during follow-up}

A total of 1576 patients were treated for a first ACS, of these $554(35.2 \%)$ had STEMI, 811 (51.5\%) NSTEMI, while $211(13.4 \%)$ had a final diagnosis of UAP. Demographic characteristics of the participants are shown in table 1 . The majority of the patients were male $(69.1 \%)$, and the mean age of the study sample was 69.3 years (SD 11.9 years). The majority of the patients underwent PCI $(77.5 \%, \mathrm{n}=1221)$, while the rates of patients treated by CABG $(8.1 \%, \mathrm{n}=127)$ or medical therapy only $(14.5 \%$, $\mathrm{n}=228)$ were substantially lower. Altogether, $106(6.7 \%)$ patients died within 6-month follow-up time after ACS. A considerable proportion of deaths $(n=42,39.6 \%)$ occurred among patients with reduced LVEF $(<40 \%)$. Details about the use of antithrombotic medications and invasive treatments are presented in the online supplement. Planned pharmacological therapies at discharge or at transfer are presented in online supplementary table 1.

\section{Prediction of 6-month mortality}

Cox-regression analyses revealed that both the GRACE score and LVEF were independently associated with 6-month mortality. In univariate analysis, the GRACE score associated with the risk for mortality with a HR of 1.040 (95\% CIs 1.034 to $1.046, \mathrm{p}<0.0001$ ) corresponding to a one-unit change in the GRACE score. As expected, LVEF was inversely associated with mortality with a $1 \%$ (unit) change in LVEF corresponding to a HR of 0.927 (95\% CIs 0.911 to $0.942, \mathrm{p}<0.0001$ ). The unadjusted overall mortality rate across different clinical categories of LVEF (reduced: $<40 \%$, mildly reduced: $40 \%-49 \%$ and normal: $\geq 50 \%$ ) are presented in table 2 .

After applying both variables into the same model, the results remained significant: the GRACE score HR was 1.036 (95\% CIs 1.030 to $1.042, \mathrm{p}<0.0001$ ) and the LVEF HR 0.965 (95\% CIs 0.948 to $0.982, \mathrm{p}<0.0001$ ). The changes of mortality risk related to LVEF across the continuum of LVEF values after adjusting for the GRACE score is depicted in figure 1 . There was no significant interaction between LVEF and the GRACE score $(p=0.397$ for the interaction), suggesting that the association between LVEF and mortality was dependent on patient's baseline risk as evaluated by the GRACE score.

Further adjustments for other comorbidities, such as previously suffered major health issues (history of MI, stroke, gastrointestinal bleed, anaemia, prior PCI or CABG), or prevalent conditions (cancer, valvular heart disease, diabetes or chronic obstructive pulmonary disease), did not change the adjusted association between LVEF and 6-month mortality (HR 0.967 with $95 \%$ CIs 0.951 to 0.984 , $\mathrm{p}=0.0001$ ). Additional adjustment with planned pharmacological therapies (at discharge, at transfer or before death in hospital, factors presented in online supplementary table 1) or with the type of ACS (UAP, NSTEMI or STEMI) did not result in significant change in the association $(0.960$ with $95 \%$ CIs 0.942 to 0.979 , $\mathrm{p}<0.0001$ ).

Model discrimination was assessed by the C-statistic. A model with a C-statistic $>0.75$ is considered to have meaningful discriminatory ability. The GRACE score predicted mortality well with a resulting C-statistic value of 0.833 (95\% CIs 0.795 to 0.871 ). Applying LVEF in the model, the resulting C-statistic was 0.848 (95\% CIs 0.813 to 0.883$)$. The difference in C-statistics was significant ( $\mathrm{p}=0.029$ for DeLong's test evaluating difference in C-statistic for paired samples).

Estimates of continuous reclassification value showed a benefit in improving the current model: IDI $0.0171(95 \%$ CIs 0.0016 to $0.0327, \mathrm{p}=0.031$ ) and NRI of 0.443 (95\% CIs 0.252 to $0.635, \mathrm{p}=0.00001$ ).

\section{DISCUSSION}

According to the results of the present study, LVEF is an independent risk factor for mortality regardless of patient 
Table 1 Baseline characteristics of patients with ACS evaluated and treated invasively in Tays Heart Hospital in 2015 and 2016

\begin{tabular}{|c|c|c|c|c|}
\hline & $\begin{array}{l}\text { All } \\
(n=1576)\end{array}$ & $\begin{array}{l}\text { Dead } \\
(n=106)\end{array}$ & Survivors $(n=1470)$ & P value* \\
\hline \multicolumn{5}{|l|}{ Demographics } \\
\hline Age (years) & $69.26 \pm 11.85$ & $76.67 \pm 10.17$ & $68.73 \pm 11.79$ & $<0.001$ \\
\hline Estimated GFR* & $82.1(26.3)$ & $62.3(28.4)$ & $83.6(25.5)$ & $<0.001$ \\
\hline Men (\%) & $1089(69.1)$ & $58(54.7)$ & $1031(70.1)$ & $<0.001$ \\
\hline Hypertension, n (\%) & $963(61.1)$ & $68(64.2)$ & $895(60.9)$ & 0.048 \\
\hline Dyslipidaemia, n (\%) & $935(59.3)$ & $61(57.5)$ & $874(59.5)$ & 0.013 \\
\hline Diabetes, $\mathrm{n}(\%)$ & $389(24.7)$ & $43(40.6)$ & $346(23.5)$ & $<0.001$ \\
\hline Previous MI, n (\%) & $309(19.6)$ & $35(33.0)$ & $274(18.6)$ & $<0.001$ \\
\hline Peripheral artery disease, $\mathrm{n}(\%)$ & $119(7.6)$ & $20(18.9)$ & $99(6.7)$ & $<0.001$ \\
\hline Previous PCI, n (\%) & $320(17.8)$ & $21(19.8)$ & $261(17.8)$ & 0.594 \\
\hline Previous CABG, $n(\%)$ & $165(6.5)$ & $16(15.1)$ & $122(8.3)$ & 0.017 \\
\hline \multicolumn{5}{|l|}{ On admission data } \\
\hline Type of ACS, n (\%) & & & & $<0.001$ \\
\hline UAP & $211(13.4)$ & $4(3.8)$ & $207(14.1)$ & \\
\hline NSTEMI & $811(51.5)$ & $49(46.2)$ & $762(51.8)$ & \\
\hline STEMI & $554(35.2)$ & $53(50.0)$ & $501(34.1)$ & \\
\hline Killip class, n (\%) & & & & $<0.001$ \\
\hline I & 1267 (80.4) & $45(42.5)$ & $1222(83.1)$ & \\
\hline$\|$ & $190(12.1)$ & $21(19.8)$ & $169(11.5)$ & \\
\hline III & $93(5.9)$ & $22(20.8)$ & $71(4.8)$ & \\
\hline IV & $26(1.6)$ & $18(17.0)$ & $8(0.5)$ & \\
\hline Heart rate (beats per min) & $79.11 \pm 20.53$ & $86.02 \pm 22.71$ & $78.63 \pm 20.29$ & $<0.001$ \\
\hline Systolic blood pressure (mm Hg) & $144.11 \pm 29.04$ & $128.27 \pm 31.84$ & $145.22 \pm 28.51$ & $<0.001$ \\
\hline Serum creatinine level ( $\mu \mathrm{mol} / \mathrm{L})$ & $90.91 \pm 58.67$ & $120.40 \pm 71.37$ & $88.79 \pm 57.08$ & $<0.001$ \\
\hline Cardiac arrest at admission, $\mathrm{n}(\%)$ & $89(5.6)$ & $21(19.8)$ & $68(4.6)$ & $<0.001$ \\
\hline TnT elevated (>50 ng/L)‡, n (\%) & $1335(84.7)$ & $103(97.2)$ & $1232(83.8)$ & $<0.001$ \\
\hline ST-segment shift§, n (\%) & $1036(65.7)$ & $90(84.9)$ & $946(64.4)$ & $<0.001$ \\
\hline GRACE score & $119.92 \pm 33.67$ & $163.36 \pm 35.69$ & $116.79 \pm 31.29$ & $<0.001$ \\
\hline Ejection fraction & $50.61 \pm 11.004$ & $41.21 \pm 11.92$ & $51.29 \pm 10.62$ & $<0.001$ \\
\hline
\end{tabular}

Categorical variables are expressed in frequency and percentage and continuous variables are presented as mean \pm SD.

${ }^{*}$ For comparison between patients who were dead at 6 months and survivors.

†Estimated glomerular filtration rate $\left(\mathrm{mL} / \mathrm{min} / 1.73 \mathrm{~m}^{2}\right)$.

‡Exceeding the 99th percentile for general population.

$\S$ Significant ST-elevation or ST-segment depression in two consecutive leads.

ACS, acute coronary syndrome; CABG, coronary artery bypass grafting;GFR, glomerular filtration rate; GRACE, Global Registry of Acute

Coronary Events; MI, myocardial infarction; NSTEMI, non-ST-elevation myocardial infarction; $\mathrm{PCl}$, percutaneous coronary intervention; STEMI,

ST-elevation myocardial infarction; TnT, troponin t; UAP, unstable angina pectoris.

status at admission measured by the GRACE score. Adding LVEF to risk prediction with the GRACE score resulted in significant improvement in risk stratification.

To the best of our knowledge, there is only one previous study published on the possible incremental predictive value of adding LVEF to risk prediction beyond the GRACE score in patients with ACS. ${ }^{11}$ The retrospective MASCARA registry study by Abu-Assi et $a l^{11}$ of 3.960 patients treated in 2004-2005 found no incremental benefit of adding LVEF to the GRACE score in predicting 6-month mortality postdischarge or in-hospital mortality among patients with MI. The different study results might, at least partly, be explained by the many advances that have transformed the medical and invasive treatment of ACS during the last decade. In the MASCARA registry, ${ }^{11}$ the revascularisation rate by any method was only $18 \%$, which is in stark contrast with the corresponding figure of $86 \%$ in the present study. Furthermore, rather than using LVEF as a continuous variable as in the present study, LVEF was categorised in three risk categories in the 
Table 2 The unadjusted overall mortality rate and status on admission across different clinical categories of left ventricular ejection fraction (LVEF) (reduced $<40 \%$, mildly reduced $40 \%-49 \%$ and normal $\geq 50 \%$ )

\begin{tabular}{|c|c|c|c|c|}
\hline & LVEF <40\% $(n=210)$ & LVEF $40 \%-49 \%(n=357)$ & LVEF $\geq 50 \%(n=1009)$ & $P$ value \\
\hline Dead at 6 months, n (\%) & $42(20.0)$ & $31(8.7)$ & $33(3.3)$ & $<0.001$ \\
\hline Age & $71.50 \pm 12.06$ & $70.23 \pm 11.95$ & $68.45 \pm 11.70$ & $<0.001$ \\
\hline GRACE score & $144.11 \pm 34.12$ & $128.55 \pm 30.88$ & $111.83 \pm 31.30$ & $<0.001$ \\
\hline Type of ACS, n (\%) & & & & $<0.001$ \\
\hline NSTEMI & $99(47.1)$ & $156(43.7)$ & $556(55.1)$ & \\
\hline STEMI & $105(50.5)$ & $172(48.2)$ & $277(27.5)$ & \\
\hline Killip class, $n(\%)$ & & & & $<0.001$ \\
\hline I & $92(43.8)$ & $261(73.1)$ & $914(90.6)$ & \\
\hline ॥ & $62(29.5)$ & $61(17.1)$ & $67(6.6)$ & \\
\hline
\end{tabular}

ACS, acute coronary syndrome;GRACE, Global Registry of Acute Coronary Events; NSTEMI, non-ST-elevation myocardial infarction; STEMI, ST-elevation myocardial infarction; UAP, unstable angina pectoris.

MASCARA registry; this could result in diminished statistical power for detecting independent associations and incremental value of adding LVEF to the risk prediction. Supporting our findings, previous studies have shown that LVEF is an independent risk marker for mortality after ACS, even after accounting for other clinical risk factors related to comorbidities and status on admission. This was the case even after including Killip classification for heart failure, ST-segment abnormalities, cardiac enzyme levels and haemodynamic status, which are included in the GRACE score. ${ }^{891718}$

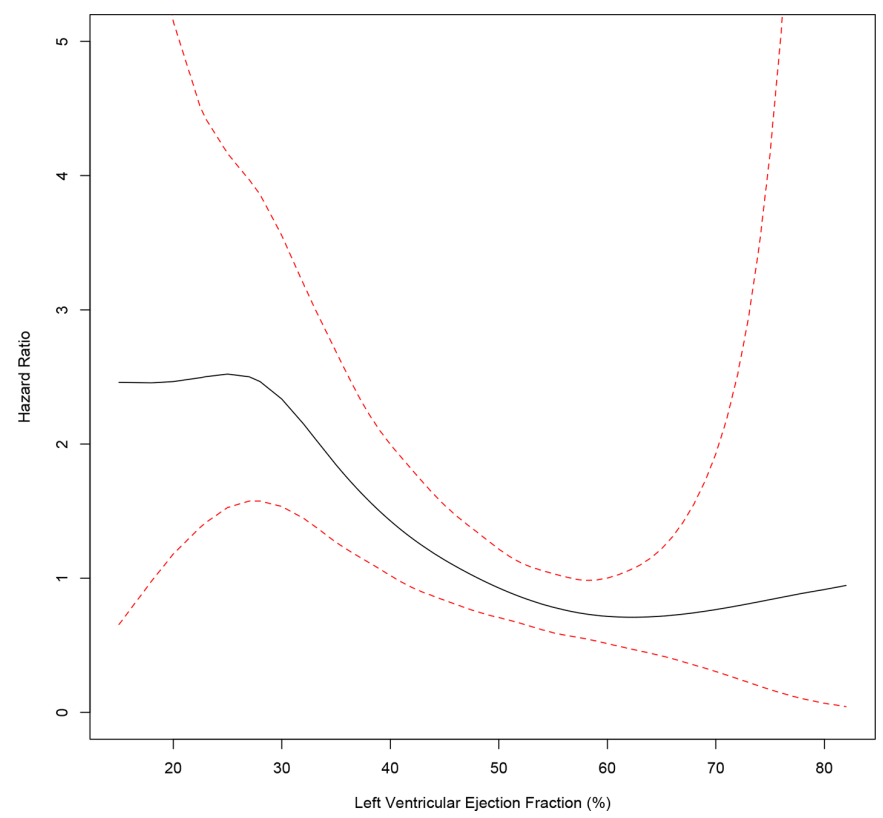

Figure 1 The association between left ventricular ejection fraction and 6-month mortality after adjusting for patients' baseline risk as evaluated by grace score depicting patients' status on admission for acute coronary syndrome.
Despite the fact that the GRACE score was introduced almost two decades ago, it still represents the best possible validated method for estimating the risk of mortality after $\mathrm{ACS},{ }^{3}$ and its use is recommended by current guidelines. ${ }^{5-7}$ After the onset of the new era of 'big data', the discovery of novel risk factors, which can add to risk prediction over clinically relevant risk markers, is important. ${ }^{19}$ Systematic recording of risk factors, such as LVEF, should be applied universally as the utilisation of registry information used to model patient survival becomes the norm. ${ }^{19}$

The limitation of the present study is that the GRACE score information was collected retrospectively and therefore was not available for all subjects. However, data were missing only for approximately $2.6 \%$ of the subjects. Retrospective collection can also be less accurate in capturing relevant information when compared with a dedicated online data collection protocol. Despite of these shortcomings, the GRACE score recorded in our study performed very well, with the C-statistic (0.83) very close to the value expected for prediction of 6-month mortality based on meta-analysis of all previous observational studies (meta-analysed C-statistic of 0.84). ${ }^{3}$ The foremost strength of the present study is that it includes all consecutive patients diagnosed and treated invasively for ACS during the time of the study. Furthermore, due to the centralised nature of Finnish national registries, there was no loss of mortality data for follow-up.

In conclusion, the GRACE score remains a good predictor of all-cause mortality in a contemporary setting. However, LVEF is an independent risk marker for death after ACS despite patient's comorbidities and status as measured by the GRACE score. LVEF can be used to improve risk prediction over the GRACE score. 
Author affiliations

${ }^{1}$ Faculty of Medicine and Health Technology, Tampere University, Tampere, Finland

${ }^{2}$ VTT Technical Research Centre of Finland, Tampere, Finland

${ }^{3}$ Tays Heart Hospital, Tampere University Hospital, Tampere, Finland

${ }^{4}$ Department of Clinical Chemistry, Fimlab Laboratories, Tampere University Hospital, Tampere, Finland

${ }^{5}$ Finnish Cardiovascular Research Center Tampere, Tampere, Finland

${ }^{6}$ Centre for Vascular Surgery and Interventional Radiology, Tampere University Hospital, Tampere, Finland

Acknowledgements TL has been supported by the Academy of Finland (grant no. 286284); Competitive State Research Financing of the Expert Responsibility area of Tampere and Turku University Hospital (grant X51001); Finnish Foundation for Cardiovascular Research; Tampere Tuberculosis Foundation; Emil Aaltonen Foundation; Yrjö Jahnsson Foundation; Signe and Ane Gyllenberg Foundation; Diabetes Research Foundation of Finnish Diabetes Association; Tampereen Yliopistollisen sairaalan tukisäätiö, and EU Horizon 2020 (grant 755320 for TAXINOMISIS). The MADDEC project has been supported by the Business Finland (the Finnish Funding Agency for Technology and Innovation).

Contributors All authors have made substantial contributions to the manuscript by drafting the work or revising it critically for important intellectual content and by participating to the design of the work, or the acquisition, analysis or interpretation of the data. The authors take full responsibility of the integrity and accuracy of the data and all authors have given their approval for the final version of the manuscript.

Funding The study was supported by the Business Finland (the Finnish Funding Agency for Technology and Innovation).

Competing interests None declared.

Patient consent for publication Not required.

Provenance and peer review Not commissioned; externally peer reviewed.

Data availability statement No data are available.

Open access This is an open access article distributed in accordance with the Creative Commons Attribution Non Commercial (CC BY-NC 4.0) license, which permits others to distribute, remix, adapt, build upon this work non-commercially, and license their derivative works on different terms, provided the original work is properly cited, appropriate credit is given, any changes made indicated, and the use is non-commercial. See: http://creativecommons.org/licenses/by-nc/4.0/.

\section{REFERENCES}

1. Naghavi M, Abajobir AA, Abbafati $C$, et al. Global, regional, and national age-sex specific mortality for 264 causes of death, 19802016: a systematic analysis for the global burden of Disease Study 2016. The Lancet 2017;390:1151-210. Sep.

2. Kumar A, Cannon CP. Acute coronary syndromes: diagnosis and management, Part I. Mayo Clin Proc 2009;84:917-38.

3. D'Ascenzo F, Biondi-Zoccai G, Moretti C, et al. TIMI, grace and alternative risk scores in acute coronary syndromes: a meta-analysis of 40 derivation studies on 216,552 patients and of 42 validation studies on 31,625 patients. Contemp Clin Trials 2012;33:507-14.

4. Fox KAA, Dabbous $\mathrm{OH}$, Goldberg RJ, et al. Prediction of risk of death and myocardial infarction in the six months after presentation with acute coronary syndrome: prospective multinational observational study (grace). BMJ 2006;333.

5. Roffi M, Patrono C, Collet J-P, et al. 2015 ESC guidelines for the management of acute coronary syndromes in patients presenting without persistent ST-segment elevation: Task Force for the management of acute coronary syndromes in patients presenting without persistent ST-segment elevation of the European Society of cardiology (ESC). Eur Heart J 2016;37:267-315.

6. Steg PG, James SK, Atar D, et al. ESC guidelines for the management of acute myocardial infarction in patients presenting with ST-segment elevation. Eur Heart J 2012;33:2569-619.

7. Jneid H, Anderson JL, Wright RS, et al. 2012 ACCF/AHA focused update of the guideline for the management of patients with unstable angina/Non-ST-elevation myocardial infarction (updating the 2007 guideline and replacing the 2011 focused update): a report of the American College of cardiology Foundation/American Heart Association Task Force on practice guidelines. Circulation 2012;126:875-910.

8. Perelshtein Brezinov O, Klempfner R, Zekry SB, et al. Prognostic value of ejection fraction in patients admitted with acute coronary syndrome: a real world study. Medicine 2017;96:e6226.

9. Mukherjee JT, Beshansky JR, Ruthazer R, et al. In-hospital measurement of left ventricular ejection fraction and one-year outcomes in acute coronary syndromes: results from the immediate trial. Cardiovasc Ultrasound 2016;14.

10. van Loo HM, van den Heuvel ER, Schoevers RA, et al. Sex dependent risk factors for mortality after myocardial infarction: individual patient data meta-analysis. BMC Med 2014:12

11. Abu-Assi E, Ferreira-González I, Ribera A, et al. "Do GRACE (Global Registry of Acute Coronary events) risk scores still maintain their performance for predicting mortality in the era of contemporary management of acute coronary syndromes?". Am Heart $J$ 2010;160:826-34.

12. Reddy K, Khaliq A, Henning RJ. Recent advances in the diagnosis and treatment of acute myocardial infarction. World J Cardiol 2015;7.

13. Dagres N, Hindricks G. Risk stratification after myocardial infarction: is left ventricular ejection fraction enough to prevent sudden cardiac death? Eur Heart J 2013;34:1964-71.

14. Hernesniemi JA, Mahdiani S, Lyytikäinen LP. Cohort description for MADDEC - mass data in detection and prevention of serious adverse events in cardiovascular disease. IFMBE proceedings, 2017:1113-6.

15. Pajunen $\mathrm{P}$, Koukkunen $\mathrm{H}$, Ketonen $\mathrm{M}$, et al. The validity of the Finnish hospital discharge register and causes of death register data on coronary heart disease. Eur J Cardiovasc Prev Rehabil 2005;12:132-7.

16. Tolonen H, Salomaa V, Torppa J, et al. The validation of the Finnish hospital discharge register and causes of death register data on stroke diagnoses. Eur J Cardiovasc Prev Rehabil 2007;14:380-5.

17. Bosch $X$, Théroux $P$. Left ventricular ejection fraction to predict early mortality in patients with non-ST-segment elevation acute coronary syndromes. Am Heart J 2005;150:215-20.

18. Sutton NR, Li S, Thomas L, et al. The association of left ventricular ejection fraction with clinical outcomes after myocardial infarction: findings from the acute coronary treatment and intervention outcomes network (ACTION) Registry-Get with the guidelines (GWTG) Medicare-linked database. Am Heart J 2016;178:65-73.

19. Hemingway $\mathrm{H}$, Asselbergs FW, Danesh J, et al. Big data from electronic health records for early and late translational cardiovascular research: challenges and potential. Eur Heart $J$ 2018;39:1481-95. 